\title{
Short-Term Plasticity of Kainate Receptor-Mediated EPSCs Induced by NMDA Receptors at Hippocampal Mossy Fiber Synapses
}

\author{
Nelson Rebola, ${ }^{1,2}$ Shankar Sachidhanandam, ${ }^{1}$ David Perrais, ${ }^{1}$ Rodrigo A. Cunha, ${ }^{2}$ and Christophe Mulle ${ }^{1}$ \\ ${ }^{1}$ Laboratoire "Physiologie Cellulaire de la Synapse," Centre National de la Recherche Scientifique Unité Mixte de Recherche 5091, Bordeaux Neuroscience \\ Institute, University of Bordeaux 2, 33077 Bordeaux, France, and ${ }^{2}$ Faculty of Medicine, Institute of Biochemistry, Center for Neuroscience and Cell Biology \\ of Coimbra, University of Coimbra, 3004-504 Coimbra, Portugal
}

\begin{abstract}
Kainate receptors (KARs) are heteromeric ionotropic glutamate receptors that play a variety of functions in the regulation of the activity of synaptic networks. Little is known about the regulation of the function of synaptic KARs in the brain. In the present study, we found that a conditioning activation of synaptic NMDA receptors (NMDARs) induces short-term depression of KAR-EPSCs but not of AMPA receptor-EPSCs at synapses between mossy fibers and CA3 pyramidal cells. Short-term depression of KAR-EPSCs by synaptic NMDARs peaked at $1 \mathrm{~s}$ and reversed within $20 \mathrm{~s}$, was likely induced and expressed postsynaptically, and was homosynaptic. It depended on a rise of $\mathrm{Ca}^{2+}$ in the postsynaptic cell and on the activation of the phosphatase calcineurin that likely binds to the GluR6b (glutamate receptor subunit $6 \mathrm{~b}$ ) subunit splice variant allowing the dephosphorylation of KARs and inhibition of activity. Finally, we show in the currentclamp mode that short-term depression of KAR-EPSPs is induced by the coincident discharge of action potentials in the postsynaptic cell together with synaptic stimulation. Hence, this study describes a form of short-term synaptic plasticity that is postsynaptic, depends on the temporal order of presynaptic and postsynaptic spiking, and likely affects the summation properties of mossy fiber EPSPs.
\end{abstract}

Key words: kainate receptors; short-term synaptic plasticity; NMDA receptors; mossy fiber; hippocampus

\section{Introduction}

Glutamatergic synaptic transmission is mediated by AMPA receptors (AMPARs) and NMDA receptors (NMDARs) that are found colocalized in the postsynaptic density of the vast majority of glutamatergic synapses in the brain and are activated concomitantly after release of glutamate from the presynaptic terminal. Postsynaptic kainate receptors (KARs) show restricted cellular and subcellular expression. Kainate receptor-mediated EPSCs (KAR-EPSCs) have been best characterized at synapses between mossy fibers and CA3 pyramidal cells in the hippocampus (Castillo et al., 1997; Vignes and Collingridge, 1997; Mulle et al., 1998; Contractor et al., 2003), although they have also been observed in a variety of other synapses (Huettner, 2003; Lerma, 2003; Pinheiro and Mulle, 2006). A single stimulation of mossy fibers is sufficient to activate synaptic KARs (Castillo et al., 1997), which only yields EPSCs of small amplitude and slow kinetics. The analysis of KAR mutant mice has further demonstrated that 1-(4-aminophenyl)-4-methyl-7,8-methylenedioxy-5H-2,3benzodiazepine (GYKI 53655)-resistant EPSCs were mediated by

Received Aug. 7, 2006; revised Feb. 12, 2007; accepted Feb. 15, 2007.

This work was supported by Fundaçao para a Ciência e para a Tecnologia Grant POCI/NEU-SAU/59135/2004, by the Centre National de la Recherche Scientifique, the Ministère de la Recherche of France, the Conseil Régional d'Aquitaine, the Fondation pour la Recherche Médicale (fellowship to S.S.), and the European Commission (EUSynapse, contract number LSH-2004-019055 to C.M.). We thank Paulo Pinheiro for criticial reading of this manuscript Correspondence should be addressed to Christophe Mulle at the above address. E-mail: mulle@u-bordeaux 2.fr. DOI:10.1523/JNEUROSCI.5182-06.2007

Copyright $\odot 2007$ Society for Neuroscience $\quad 0270-6474 / 07 / 273987-07 \$ 15.00 / 0$
KARs composed of the glutamate receptor subunit 6 (GluR6) and KA2 subunits (Mulle et al., 1998; Contractor et al., 2003; Ruiz et al., 2005).

Compared with AMPA and NMDA receptor-mediated synaptic transmission, little is known about the plasticity of KAREPSCs. Early during postnatal development, long-term potentiation expression at thalamocortical synapses is associated with a reduction of KAR-EPSCs (Kidd and Isaac, 1999). In perirhinal cortex neurons, activation of PKC by stimulation of mGluR5 enhances KAR-EPSCs (Cho et al., 2003; Park et al., 2006). In these neurons, a train of 200 stimuli at $5 \mathrm{~Hz}$ results in long-term depression of KAR-EPSCs via a mechanism involving mGluR5, PKC, and PICK1 (protein interacting with C kinase-1) PDZ (postsynaptic density-95/Discs large/zona occludens-1) domain interactions (Park et al., 2006), and possibly a change in the number of synaptic KARs (Hirbec et al., 2003). At hippocampal mossy fiber synapses, KAR-EPSCs are regulated as a consequence of presynaptic changes in glutamate release properties (Ito et al., 2004), but no form of synaptic plasticity specific for KAR-EPSCs has yet been described. However, the functional properties of recombinant KARs can be regulated on a shorter time scale by direct cAMP-dependent phosphorylation (Traynelis and Wahl, 1997). In cultured hippocampal neurons, a conditioning activation of NMDARs depresses native KAR-mediated responses in a rapid and reversible manner (Ghetti and Heinemann, 2000). This depression depends on $\mathrm{Ca}^{2+}$ influx through NMDA receptor or voltage-gated calcium channels and on the activation of cal- 
cineurin (Ghetti and Heinemann, 2000). It also requires the heteromeric assembly of GluR6a and GluR6b and the association of calcineurin with the C-terminal domain of GluR6b in the KAR complex (Coussen et al., 2005). The present experiments were designed to examine whether synaptic KARs at hippocampal mossy fiber synapses were prone to a phos-phorylation-dependent regulation and to define whether this resulted in postsynaptic plasticity of KAR-EPSCs.

\section{Materials and Methods}

Transverse hippocampal slices (350 $\mu \mathrm{m}$ thick) were obtained from 15 - to 21 -d-old C57BL/6 mice. Slices were transferred to a recording chamber and continuously superfused with an oxygenated extracellular medium $\left(95 \% \mathrm{O}_{2}\right.$ and $5 \% \mathrm{CO}_{2}$ ) containing (in $\mathrm{mm}$ ): $125 \mathrm{NaCl}, 2.5$ $\mathrm{KCl}, 2 \mathrm{CaCl}_{2}, 0.6 \mathrm{MgCl}_{2}, 1.25 \mathrm{NaH}_{2} \mathrm{PO}_{4}, 26$ $\mathrm{NaHCO}_{3}, 16$ glucose, pH 7.4. Whole-cell voltage-clamp recordings were made at room temperature from CA3 pyramidal cells. The intracellular solution contained the following (in $\mathrm{mm}$ ): 140 cesium methanesulfonate, $2 \mathrm{MgC}_{2}, 4$ $\mathrm{NaCl}, 5$ phospho-creatine, $2 \mathrm{Na}_{2} \mathrm{ATP}, 1$ EGTA, 10 HEPES, 0.33 GTP, pH 7.3. For currentclamp recordings, the following intracellular solution was used (in mM): 130 potassium gluconate, $2 \mathrm{MgCl}_{2}, 4 \mathrm{NaCl}$, 5 phospho-creatine, 2 $\mathrm{Na}_{2}$ ATP, 1 EGTA, 10 HEPES, 0.33 GTP, pH 7.3. In some experiments, $1 \mathrm{~mm}$ EGTA was replaced by $20 \mathrm{~mm}$ BAPTA, and in others, $100 \mu \mathrm{M}$ peptide corresponding to the last 15 aa of GluR6b or to a 15 aa scramble sequence (Coussen et al., 2005) was added to the intracellular solution together with a cocktail of anti-proteases (pepstatin, aprotinin, leupeptin, and pefabloc, $10 \mu \mathrm{g} / \mathrm{ml}$ each). Neurons were voltage clamped at $-80 \mathrm{mV}$, unless otherwise indicated, and bicuculline $(10 \mu \mathrm{M})$ was present in the superfusate of all experiments. The access resistance was $<20 \mathrm{M} \Omega$, and results were discarded if it changed by $>20 \%$.

Mossy fiber (Mf)-EPSCs were evoked by minimal intensity stimulation (Marchal and Mulle, 2004) and were characterized by a marked facilitation after switching stimulus frequency from 0.1 to $1 \mathrm{~Hz}$ and by their sensitivity to group $2 / 3$ mGluR agonist LCCG-1 [(2S,1S,2S)-2(carboxycyclopropyl)glycine]

$(10 \mu \mathrm{M})$. Recordings were made using an EPC 9 amplifier (HEKA Elektronik, Lambrecht/Pfalz, Germany) and were filtered at $0.5-1 \mathrm{kHz}$, digitized at $5 \mathrm{kHz}$, and stored on a personal computer for additional analysis (IGOR PRO 5.0; WaveMetrics, Lake Oswego, OR). Values are presented as mean \pm SEM. Either a paired or unpaired Student's $t$ test was used to define statistical differences between values.

All drugs were obtained from Tocris Bioscience (Ballwin, MO) or Sigma (St. Louis, MO). SR141716A [N-piperidino-5-(4-chlorophenyl)-1-(2,4dichlorophenyl)-4-methyl-pyrazole-3-

carboxamide] was kindly provided by Olivier Manzoni (INSERM, University of Bordeaux 2).

\section{Results}

We examined the possibility that synaptic NMDARs could regulate the function of KARs at synapses between mossy fibers and CA3 pyramidal cells. Mf-EPSCs were evoked in CA3 pyramidal cells at minimal stimulation intensity in the presence of bicuculline $(10 \mu \mathrm{M})$ to block $\mathrm{GABA}_{\mathrm{A}}$ receptors, and unless otherwise indicated, GYKI $53655(50 \mu \mathrm{M})$ to block AMPARs. Under these

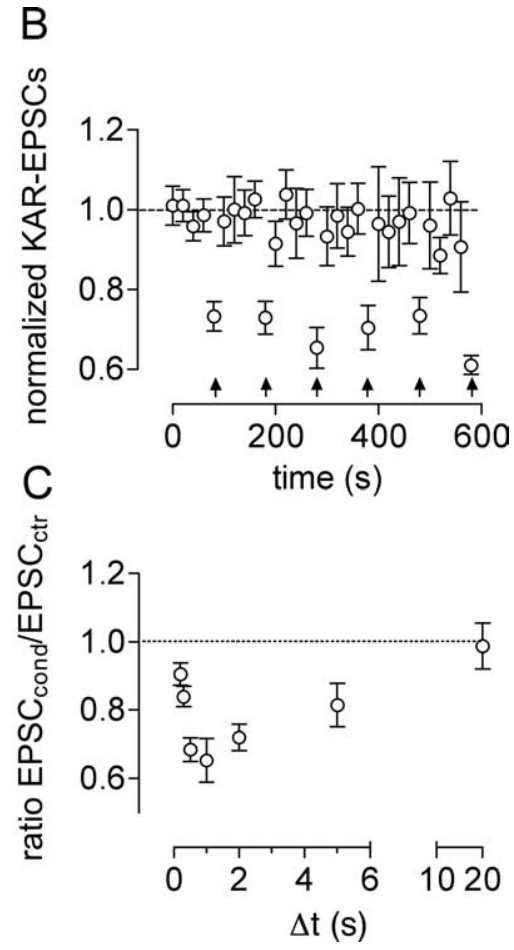

Figure 1. Short-term depression of KAR-EPSCs at mossy fiber synapses. A, Representative traces illustrating short-term depression of mossy fiber KAR-EPSCs in CA3 pyramidal cells. A train of five stimulation pulses at $20 \mathrm{~Hz}$ was delivered to mossy fibers, by a test stimulation ( $\Delta t=500 \mathrm{~ms}$ ). The train was either given with the cell membrane potential being held at $-80 \mathrm{~m}$ 列

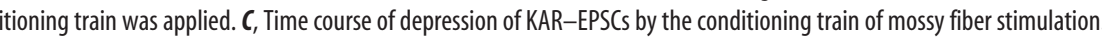

conditions (with $0.6 \mathrm{mM} \mathrm{Mg}^{2+}$ in the extracellular medium), Mf-EPSCs recorded at $-80 \mathrm{mV}$ were blocked ( $>95 \%$ inhibition) by CNQX (25 $\mu \mathrm{M}$; data not shown). In GluR6 ${ }^{-1-}$ mice in which KAR-EPSCs are abolished (Mulle et al., 1998), residual MfEPSCs of small amplitude could be recorded at $-80 \mathrm{mV}$. These residual EPSCs that corresponded to the NMDA receptor component of the Mf synaptic response only represented $7.5 \pm 1.8 \%$ $(n=8)$ of the amplitude of Mf-EPSCs recorded in wild-type mice. Thus, at this membrane potential in the presence of GYKI 53655, Mf-EPSCs are in great part mediated by KARs. In response to a train of five pulses at $20 \mathrm{~Hz}$, the amplitude of KAREPSCs during the train increased and remained potentiated 500 $\mathrm{ms}$ after the train because of presynaptic facilitation (Salin et al., 1996; Ito et al., 2004) (Fig. 1A).

To examine the consequences of synaptic activation of NMDARs on KAR-EPSCs, we depolarized the membrane to -40 $\mathrm{mV}$ while applying the train of five stimulation pulses at $20 \mathrm{~Hz}$ (conditioning train). The membrane potential was set back to $-80 \mathrm{mV} 50 \mathrm{~ms}$ after the last stimulus in the train, and a test stimulus was given $500 \mathrm{~ms}$ thereafter (Fig. $1 \mathrm{~A}$, bottom). At -40 $\mathrm{mV}, \mathrm{Mf}-\mathrm{EPSCs}$ were only inhibited by $25 \pm 10 \%(n=3)$ by CNQX, indicating that NMDARs largely contribute to MfEPSCs under these conditions. We compared the amplitude of KAR-EPSCs in response to a test stimulus given $500 \mathrm{~ms}$ after a control train (membrane potential held at $-80 \mathrm{mV}$ ) or after a conditioning train. The conditioning train led to the decrease of KAR-EPSC amplitude by $32 \pm 3 \%(n=11)$ (Fig. $1 A, B)$. The depression of KAR-EPSCs was transient and highly reproducible 
during repetition of the conditioning train (Fig. $1 B$ ). Depolarization of the cell membrane to $-40 \mathrm{mV}$ in the absence of synaptic stimulation did not significantly affect the amplitude of KAREPSCs (102 $\pm 6 \%$ of control; $n=8)$. By varying the interval between the train and the test stimulus, we observed that depression of KAR-EPSCs by a conditioned train developed progressively from almost no effect at $0.2 \mathrm{~s}$ to a peak at $1 \mathrm{~s}$ (Fig. 1C). KAR-EPSCs returned to control levels with an interval of $20 \mathrm{~s}$. Changing the parameters of the conditioning protocol by increasing the number of stimulation pulses to 10 or increasing the rate of stimulation within the train to $50 \mathrm{~Hz}$ did not further increase the extent or time course of KAR-EPSC depression (10 stimulation pulses at $20 \mathrm{~Hz}, 25 \pm 4 \%, n=7 ; 5$ stimulation pulses at $50 \mathrm{~Hz}, 28 \pm 2 \%, n=4)$.

Short-term depression of KAR-EPSCs requires changes in the membrane potential of the postsynaptic neuron. This could result in changes in the properties of postsynaptic KARs. Alternatively, depression could be induced postsynaptically and expressed presynaptically through the release of a retrograde messenger, such as what is observed for depolarization-induced suppression of excitation (Diana and Marty, 2004). In this case, depression should affect similarly both AMPAR- and KARmediated EPSCs. We thus examined the effect of a conditioning train on AMPAR-EPSCs (in the absence of GYKI 53655) (Fig. $2 A$ ). A control train induced post-tetanic potentiation of AMPAR-EPSCs (increase by $434 \pm 81 \%, n=8$ ) when comparing the first EPSC in the train and the test EPSC (EPSC $\left.C_{\text {ctr }}\right)$. The amplitude of the test AMPAR-EPSC was not significantly different $(p>0.05)$ in response to a conditioning train (Fig. $2 A, B)$, indicating that the conditioning protocol selectively affects KAR function and strongly suggesting that the mechanisms of depression do not involve an alteration of glutamate release. In support of this notion, pharmacological manipulations that are known to increase or decrease mossy fiber synaptic transmission at a presynaptic level did not affect the extent of KAR-EPSC depression (supplemental Fig. 1, available at www.jneurosci.org as supplemental material). For instance, activation of adenosine $A_{1}$ receptors tonically depresses Mf-EPSCs by acting at a presynaptic level (Nicoll and Schmitz, 2005). Accordingly, bath application of the $\mathrm{A}_{1}$ receptor antagonist 8-cyclopentyl-1,3-dipropylxanthine (100 nM) increased Mf-EPSC amplitude (153 $\pm 17 \%$ of control MfEPSC amplitude; $n=5$ ), without affecting the extent of depression of KAR-EPSCs $(29 \pm 5 \% ; n=5)$. Conversely, the $\mathrm{A}_{1}$ receptor agonist CADO $(1 \mu \mathrm{M})$ decreased Mf-EPSC amplitude (to $39 \pm 9 \%$ of control Mf-EPSC amplitude; $n=7$ ) but did not change the depression of KAR-EPSCs $(30 \pm 3 \% ; n=7)$. Finally, forskolin $(10 \mu \mathrm{M})$, which is known to increase mossy fiber synaptic transmission by acting at a presynaptic level (Nicoll and Schmitz, 2005) (236 \pm 15 of control Mf-EPSC amplitude; $n=4)$, did not affect the depression of KAR-EPSCs $(27 \pm 5 \% ; n=7)$.

Short-term depression of KAR-EPSCs was not observed in conditions in which NMDARs were blocked by D-AP5 $(50 \mu \mathrm{M})$ (depression by $7 \pm 2 \%, n=7$ ) and in conditions in which intracellular $\mathrm{Ca}^{2+}$ rise in the postsynaptic cell was prevented by the fast $\mathrm{Ca}^{2+}$ chelator BAPTA ( $20 \mathrm{~mm}$ ) (depression of KAR-EPSC by $3 \pm 6 \% ; n=4)$ (Fig. $2 B$ ). It was reported that NMDA application on cultured neurons decreases KAR-mediated inward currents by the activation of the phosphatase calcineurin (Ghetti and Heinemann, 2000). When the calcineurin inhibitor deltamethrin $(500 \mathrm{nM})$ was included in the recording pipette, the conditioning protocol did not significantly affect KAR-EPSCs (depression by $1 \pm 6 \% ; n=6$ ) (Fig. $2 B$ ). Recovery from depression was strongly reduced when the calcium/calmodulin-dependent protein kinase
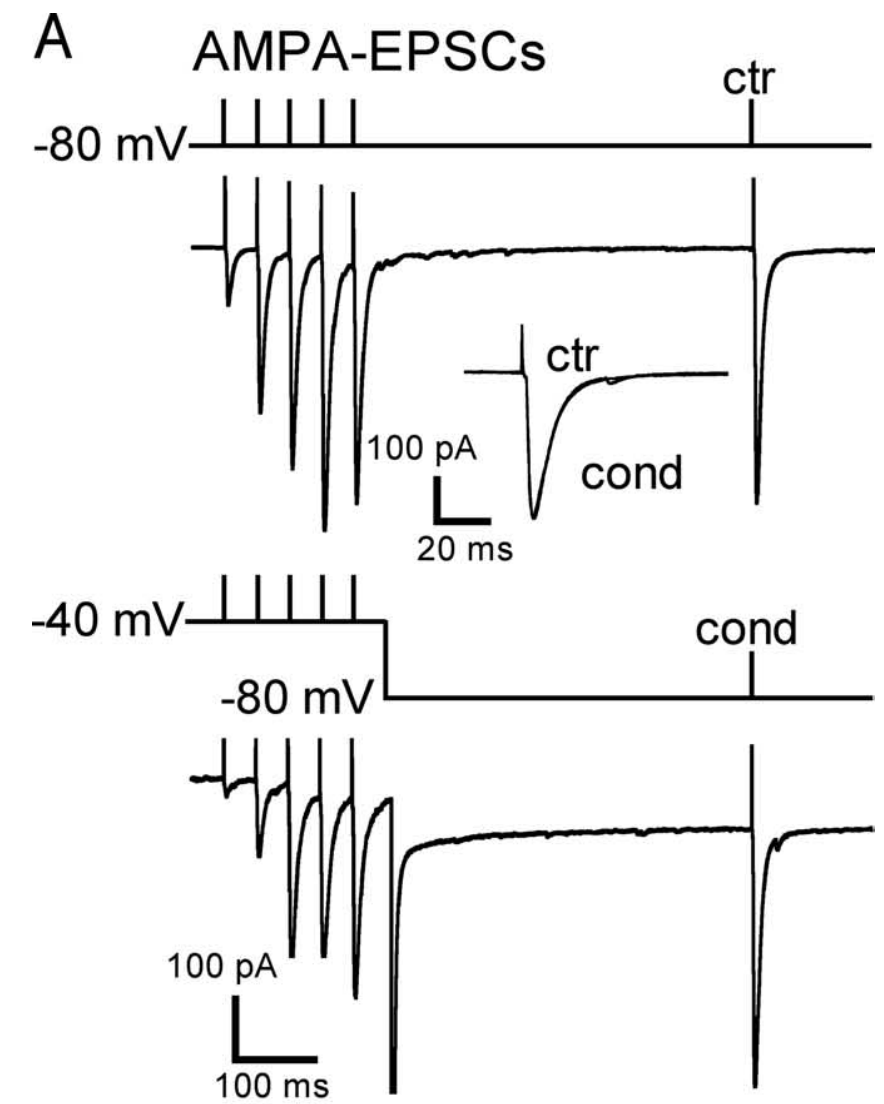

B

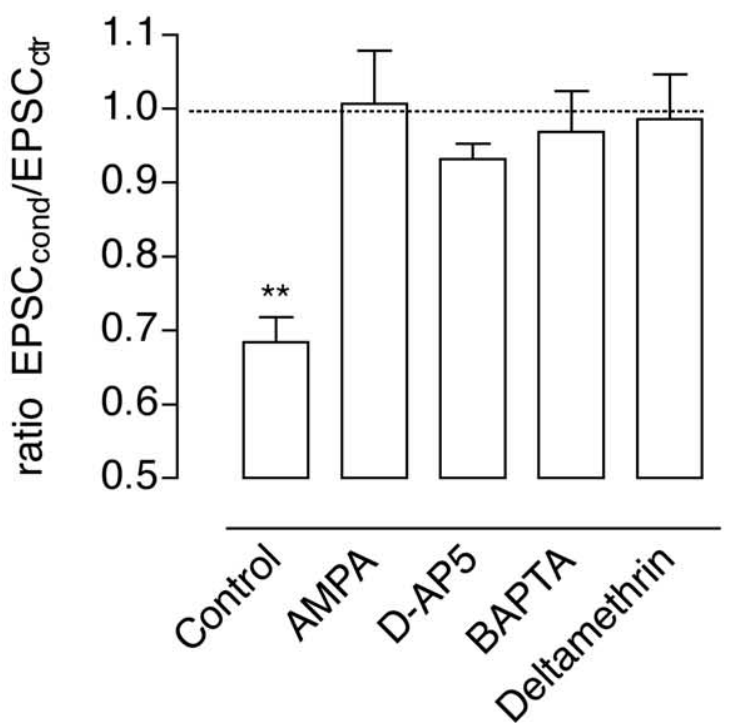

Figure 2. Short-term depression of KAR-EPSCs involves NMDA activation, postsynaptic calcium rise, and calcineurin activation. $A$, Effects of the conditioning train on AMPAR-EPSCS. Enlarged traces for $\mathrm{EPSC}_{\mathrm{ctr}}$ and $E P S \mathrm{C}_{\text {cond }}$ are superimposed in inset. ctr, Control train; cond, conditioning train. $\boldsymbol{B}$, Summary of the effects of the conditioning train in different experimental conditions. The conditioning train depressed KAR-EPSCS (Control), but not AMPAR-EPSCs, and was abolished in presence of the NMDAR antagonist D-AP5 $(50 \mu \mathrm{M})$, or when BAPTA $(20 \mathrm{~mm})$ or the phosphatase calcineurin inhibitor deltamethrin $(100 \mu \mathrm{M})$ were included in the patch pipette. ${ }^{* *} p<0.01$

II (CaMKII) antagonist KN-62 (50 $\mu \mathrm{M})$, was present in the patch pipette (at $\Delta t=20 \mathrm{~s}$ after the train of stimulation, depression of KAR-EPSC by $21 \pm 7 \% ; n=4)$. Thus, synaptic activation of NMDARs leads to short-term depression of KAR-EPSCs that 
appears to depend on the influx of $\mathrm{Ca}^{2+}$ in the postsynaptic pyramidal cell and on the activation of the phosphatase calcineurin.

We recently proposed that binding of calcineurin to GluR6b was necessary for the inhibition of kainate-evoked currents by activation of NMDARs in cultured hippocampal neurons (Coussen et al., 2005). Assuming that postsynaptic KARs at mossy fiber synapses might be composed of both GluR6a and GluR6b, we tested whether GluR6b was also determinant for the NMDAR-induced short-term depression of KAR-EPSCs. We first tested the effects of blocking GluR6b interaction with calcineurin by inclusion of a GluR6b C-terminal peptide (pepR6b) (Coussen et al., 2005) in the recording pipette (Fig. $3 A$ ). Dialysis of pepR6b in CA3 pyramidal cells did not affect the average amplitude of KAR-EPSCs during the time of the experiment (Fig. 3C,D). Short-term depression of KAR-EPSCs by a conditioning train was significantly attenuated in the presence of pepR6b (Fig. 3A,E) (depression by $11 \pm 3 \% ; n=8 ; p<0.05)$ but not in the presence of a scramble peptide (see experimental procedures) (depression of $30 \pm 2 \% ; n=6$ ) (Fig. $3 E$ ). We also tested the effects of synaptic NMDAR activation on KAR-EPSC depression in mice expressing GluR6a but not GluR6b. For this, we used transgenic mice that express mycGluR6a in hippocampal neurons on a GluR6 $^{-1-}$ background (Coussen et al., 2002). As previously shown, the mycGluR6a transgene rescued the loss of functional synaptic KARs that is observed in GluR6 $^{-1-}$ mice (Fig. 3B). The conditioning train failed to induce a significant depression of KAR-EPSCs in CA3 pyramidal cells from mycGluR6a $\times$ GluR6 $^{-/-}$mice $(9 \pm 9 \%, n=6)$ (Fig. $3 B, E)$. This set of experiments lends additional support to the notion that binding of calcineurin to the C-terminal domain of GluR6b subunit is essential for phosphatase activity on the KAR complex that leads to short-term depression of KAR-EPSCs.

From a functional point of view, it is important to understand whether short-term depression is homosynaptic or heterosynaptic. For this, we stimulated with minimal intensity two independent mossy fiber synaptic pathways by using two electrodes positioned in distinct regions of the dentate granule cell region. This experimental setup provides conditions for maximizing the likelihood that the two mossy fiber pathways were independent. As a confirmation, we checked that there was no cross-potentiation between the two synaptic inputs stimulated (stim 1 or stim 2) as expected for two independent inputs: stimulation of input 1 did not cause any increase in the amplitude of Mf-EPSCs triggered by stimulation of input 2 (and vice versa) (Fig. $4 A$ ). A conditioning train to the first stimulating electrode (stim 1) applied at $-40 \mathrm{mV}$ did not induce any significant depression of KAR-EPSCs evoked
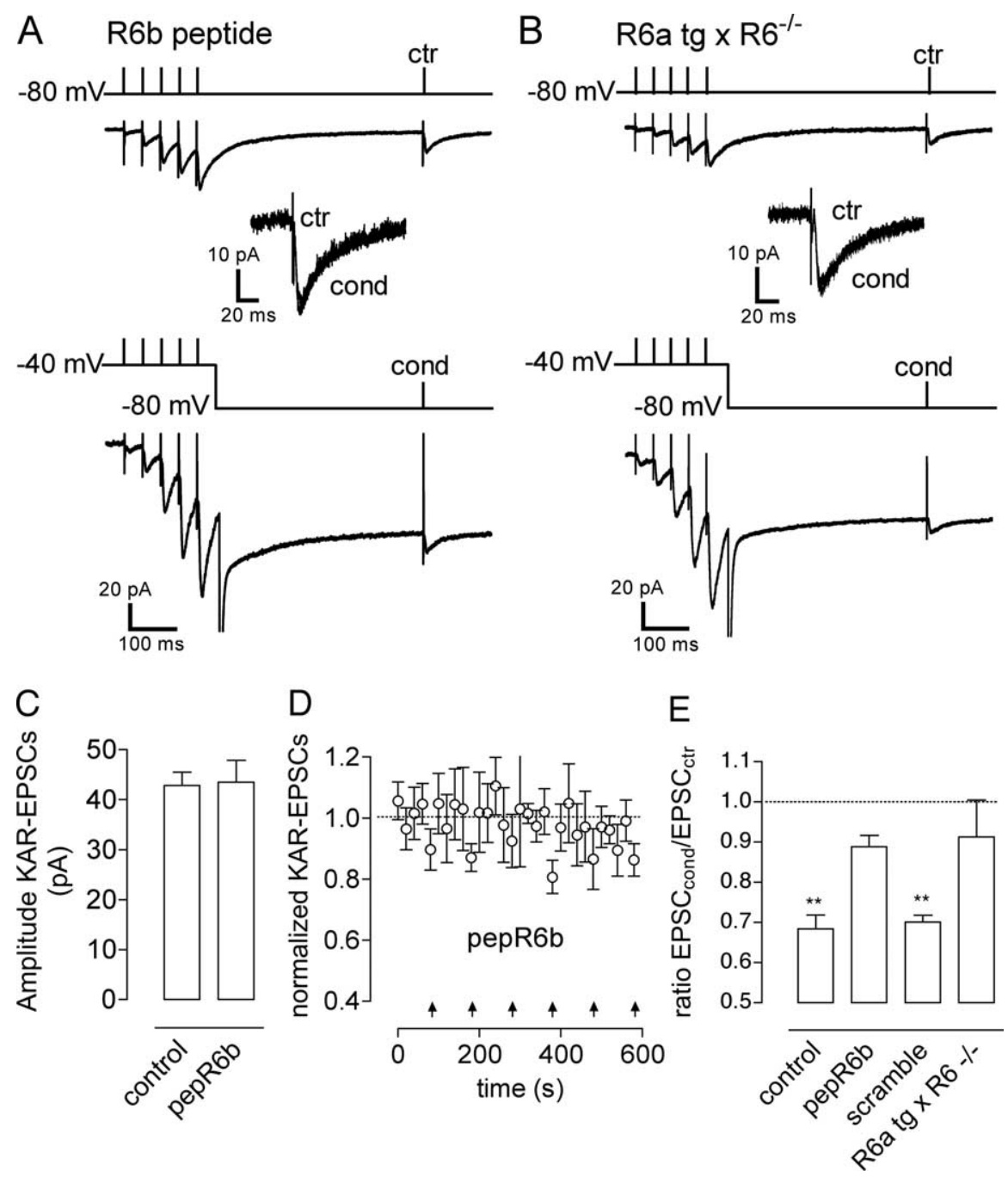

Figure 3. GluR6b subunit is necessary for NMDA-induced short-term depression of KAR-EPSCs. $\boldsymbol{A}$, Representative traces illustrating short-term depression of mossy fiber KAR-EPSCs in CA3 pyramidal cells obtained when a GluR6b C-terminal peptide ( pepR6b), which blocks the GluR6b interaction with calcineurin, was included in the patch pipette. $B$, Effects of the conditioning in the recording pipette, but not its scramble version, or working with slices from R6a $\mathrm{tg} \times \mathrm{R}^{-1-}$ mice greatly

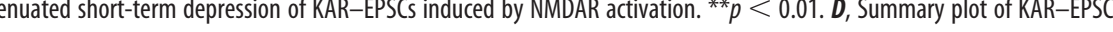
course of normalized mossy fiber KAR-EPSCs obtained when the pepR6b was present in the patch pipette. The KAR-EPSC value recording. Arrows indicate the time when the conditioning train was applied. The presence of the pepR6b in the patch pipette did not affect the time course of control KAR-EPSCs, but it strongly reduced the depression induced by the conditioning train.

in response to the second stimulating electrode (stim 2) (depression by $1 \pm 7 \%$; $n=8$ ) (Fig. $4 B$, right, $C$ ), whereas a similar conditioning protocol in which both the conditioning and the test stimuli were applied to the same mossy fiber pathway led to the depression of KAR-EPSCs (Fig. $4 B$, left, $C$ ). These data indicate that short-term depression of KAR-EPSCs by synaptic NMDARs can be induced homosynaptically and thus requires the close localization of these two receptor populations.

To address the physiological relevance of this phenomenon, we performed experiments in the current-clamp mode. Because influx of $\mathrm{Ca}^{2+}$ through NMDARs is needed for short-term depression of KAR-EPSCs, we used a conditioning protocol whereby synaptic NMDARs are relieved from the $\mathrm{Mg}^{2+}$ block through transient depolarization applied in a timely manner with 
A

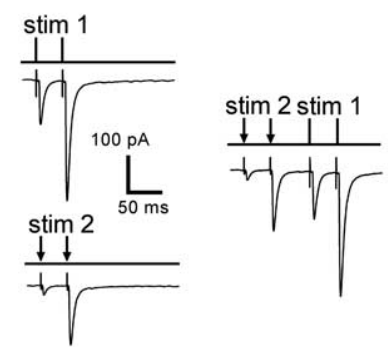

B
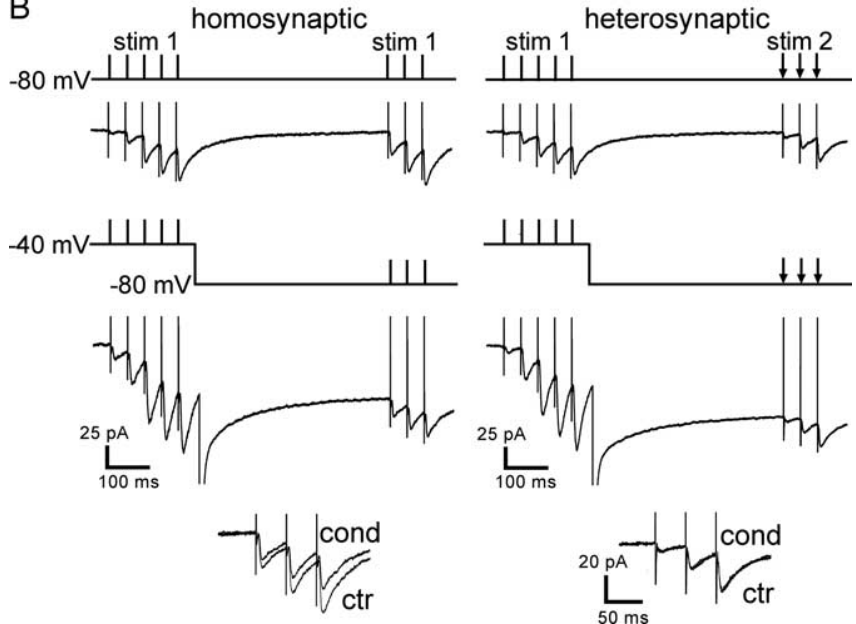

Figure 4. Short-term depression of KAR-EPSCs is homosynaptic. $\boldsymbol{A}$, Representative traces of mossy fiber-EPSCs illustrating the protocol used to verify whether two independent mossy fibers were being stimulated. Two glass stimulating electrodes were positioned at different loci in the dentate gyrus [stimulation (stim) 1 and stim 2]. Prominent paired-pulse facilitation is observed for both mossy fiber synaptic inputs applied independently (stim 1 and stim 2). However, no potentiation to stim 1 is observed after stim 2 (and vice versa). The two fibers stimulated by stim 1 and stim 2 were considered to be independent. $\boldsymbol{B}$, Homosynaptic: the conditioning protocol was applied to the same fiber as the test stimulation leading to short-term depression of KAR-EPSCS. Heterosynaptic: for two independent stimulations, applying the conditioning train to stim 1 was without any effect on KAR-EPSCs evoked in response to stim 2 . The corresponding superimposed traces are enlarged in inset. ctr, Control train; cond, conditioning train. C, Summary plot of the results obtained when stimulating two different mossy fibers, testing whether applying the conditioning protocol to one mossy fiber could also induce the short-term depression of KAR-EPSCs recorded from stimulation of a second and independent mossy fiber. ${ }^{*} p<0.05$.

the synaptic release of glutamate. For this, we paired mossy fiber stimulation to a short depolarizing pulse that triggered action potentials ( $2 \mathrm{~ms}, 800 \mathrm{pA}$ ) given $10 \mathrm{~ms}$ after each mossy fiber stimulation in the train. The depolarization resulting from the action potential during activation of NMDARs by synaptically released glutamate will largely favor the opening of NMDAR channels. We recorded a test EPSP $2 \mathrm{~s}$ after this conditioning train (at this point the membrane potential was on average $-66.7 \pm$ $2.9 \mathrm{mV}, n=7$, in control and $-66.8 \pm 2.8 \mathrm{mV}, n=7$, after a conditioning train). This pairing protocol decreased the amplitude of the test KAR-EPSP compared with a control train (no pairing) (depression by $21 \pm 5 \% ; n=7 ; p<0.05$ ) (Fig. $5 A, C$ ). In contrast, if the short depolarizing pulse was given $10 \mathrm{~ms}$ before depolarization, no change in the amplitude of the test KAR-EPSP was observed (Fig. 5B,C). Depression of KAR-EPSPs was prevented when BAPTA $(20 \mathrm{~mm})$ was included in the recording pipette (depression by $5 \pm 2 \% ; n=6$ ) and was inhibited by D-AP5 ( $3 \pm 4 \%, n=11$ ) (Fig. 5B). Thus, coincidence between action potential discharge and synaptic stimulation at mossy fi- ber synapses leads to short-term plasticity of KAR-EPSPs through activation of synaptic NMDARs.

Although a short train of action potentials alone (five spikes) was ineffective in triggering short-term depression of KAREPSPs, influx of $\mathrm{Ca}^{2+}$ through voltage-gated channels after a much larger train of action potentials (30 spikes) similarly led to the depression of KAR-EPSPs (depression by $31 \pm 2 \%$; $n=3$ ) (Fig. $6 B, C$ ) in an NMDA-independent manner (depression by $29 \pm 8 \% ; n=3$ in the presence of $50 \mu \mathrm{M}$ D-AP5). We studied the relevance of this $30 \%$ reduction of KAR-EPSPs in more physiological conditions of synaptic transmission, in the absence of the AMPA receptor antagonist GYKI 53655. We stimulated mossy fibers with a train of five stimuli and compared EPSPs obtained in control conditions versus EPSPs obtained after applying the conditioning protocol that consisted of a train of 30 spikes induced by current injection, before synaptic stimulation. This conditioning protocol did not change the amplitude of the first EPSP in the train (Fig. $6 D, E$ ), suggesting that the train of 30 spikes did not have any presynaptic effects, nor did it affect AMPA receptors. However, we observed a clear change in the waveform of MfEPSPs evoked by a train of five stimuli (Fig. 6D). The kinetic difference is subtle for a single Mf-EPSP, but for subsequent stimuli, a decrease in the decay of Mf-EPSPs is observed that changes summation properties likely attributable to the $30 \%$ decrease in KAR-EPSC amplitude. After four stimuli, summation of Mf-EPSPs is significantly decreased (decrease of $37 \pm 7 \%$; $n=$ 6) (Fig. 6D, parameter "a," $F$ ), leading in several instances to a difference in the capacity to trigger action potentials (data not shown). This effect was not attributable to the calciumdependent inactivation of NMDA receptors (Tong et al., 1995) because we could observe the same decrease in Mf-EPSPs summation in the presence of D-AP5 (decrease of $40 \pm 5 \%$; $n=5$; data not shown).

\section{Discussion}

Short-term changes in synaptic strength are commonly thought to be mediated by presynaptic mechanisms such as $\mathrm{Ca}^{2+}$ control of release probability or presynaptic vesicle replenishment (Zucker and Regehr, 2002). Here we describe a form of shortterm plasticity of KAR-EPSCs at mossy fiber synapses that is induced and expressed postsynaptically, in response to the coincident activation of NMDARs during a short burst of afferent mossy fiber stimulation.

Several lines of evidence, such as the requirement for the activity of postsynaptic NMDARs and $\mathrm{Ca}^{2+}$ increase in the postsynaptic CA3 pyramidal cell, argue in favor of a postsynaptic induction mechanism for short-term depression of KAR-EPSCs. Nevertheless, it could be argued that short-term plasticity of KAR-EPSCs is expressed presynaptically. Indeed, postsynaptic depolarization (possibly in conjunction with activation of postsynaptic NMDARs) can release retrograde messengers, such as endocannabinoids, that can act presynaptically to cause shortterm depression of transmitter release (Alger, 2002; Diana and Marty, 2004). We do not favor this type of mechanism for several reasons. First, short-term depression does not affect AMPAREPSCs, as would be expected if glutamate release was involved in the short-term depression of KAR-EPSCs. In addition, pharmacological manipulations that alter Mf-EPSCs (including KAREPSCs) by acting at a presynaptic level are without any effect on the extent of short-term depression of KAR-EPSCs. Finally, short-term depression of KARs by synaptic NMDARs is not observed in transgenic mice with postsynaptic KARs containing GluR6a and not GluR6b, as well as with a postsynaptic infusion of 
the GluR6b peptide in pyramidal cells. Thus, this form of short-term depression of synaptic transmission is both induced and expressed postsynaptically.

We propose that the mechanism of short-term depression of KAR-EPSCs involves the $\mathrm{Ca}^{2+}$-dependent activation of the phosphatase calcineurin. Previous studies have shown that kainate-activated currents in hippocampal cultured neurons are depressed by NMDARs and by voltagedependent $\mathrm{Ca}^{2+}$ channels through activation of calcineurin (Ghetti and Heinemann, 2000). We give additional evidence for such a mechanism under physiological conditions of activation of synaptic NMDARs. Our experimental protocol allows to finely detail the time course of inhibition of KAREPSCs, which appears to develop progressively over the first second after NMDAR activation and to recover within $20 \mathrm{~s}$. This time course is reminiscent of the calcineurindependent regulation of desensitization of synaptic NMDARs (Tong et al., 1995), which can thus regulate their own activity as well as the activity of KARs. It appears that $\mathrm{Ca}^{2+}$ influx through the KAR channel itself is not sufficient to activate the calciumdependent enzyme calcineurin, possibly because of the low calcium permeability of synaptic KARs that might be predominantly edited (Castillo et al., 1997). As in hippocampal cultured neurons, the recovery from depression of KAR-EPSCs could be prevented by an inhibitor of CaMKII. Overall, these data favor a mechanism whereby synaptic KARs exist, at least partly, in a phosphorylated form and can be dephosphorylated by the calcium-dependent activation of calcineurin, leading to decreased receptor channel activity (Traynelis and Wahl, 1997). It is not yet clear whether one of the KAR subunits of postsynaptic KARs at mossy fiber synapses is prone to phosphorylation by $\mathrm{Ca}^{2+}$-calmodulin kinase II, or whether it is instead a KAR-associated protein. Nevertheless, our data lend further support to the notion that the regulation of KAR function requires the binding of calcineurin to GluR6b (Coussen et al., 2005). Because of its rapid onset and the similarity with studies on recombinant receptors (Traynelis and Wahl, 1997), which implicate a decrease in the opening probability of KAR channels, short-term depression of KAR-EPSCs is likely attributable to a decrease in the function of synaptic KARs, although the removal of KARs from the postsynaptic membrane, a mechanism suggested to occur for longterm depression of synaptic KARs in the perirhinal cortex (Park et al., 2006), cannot be excluded.

Short-term depression of synaptic KARs can occur through a local and synapse-specific mechanism requiring an increase in

A

$10 \mathrm{~ms}$ stimuli. ${ }^{*} p<0.05$.

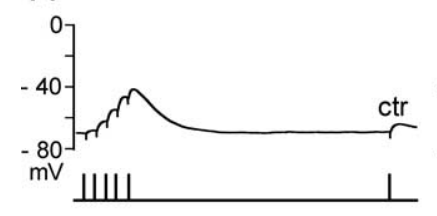

B

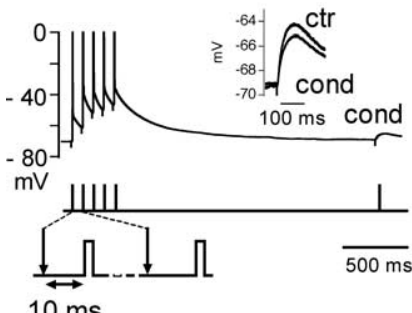

Figure 5. Pairing mossy fiber stimulation with postsynaptic depolarization depresses KAR-EPSPs. $A$, Current-clamp recordings from CA3 pyramidal cells recorded in the current-clamp mode in the presence of $1.3 \mathrm{~mm}$ extracellular $\mathrm{Mg}^{+2}$. Evoking spikes with short depolarizing pulses ( $2 \mathrm{~ms}, 800 \mathrm{pA}) 10 \mathrm{~ms}$ after mossy fiber stimulation (post depol) induced depression of KAR-EPSPs. $\boldsymbol{B}$, If the depolarizing pulses were each applied $10 \mathrm{~ms}$ before mossy fiber stimulation (pre depol), no significant effect of the protocol on KAR-EPSPs was observed. ctr, Control train; cond, conditioning train. $\boldsymbol{C}$, Summary of the effects of the protocol used in $\boldsymbol{A}$ on KAR-EPSPs in control conditions, in conditions in which $20 \mathrm{~mm}$ BAPTA was included in the patch pipette or after inhibition of NMDARs with $50 \mu \mathrm{M}$ D-AP5, and with the conditions used in $\boldsymbol{B}$. ${ }^{*} p<0.05$
A

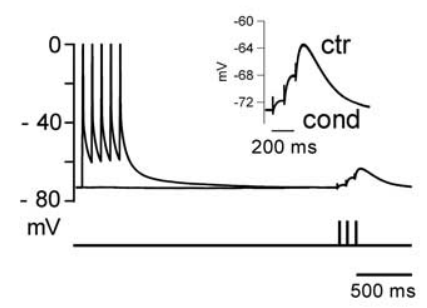

$\mathrm{D}$

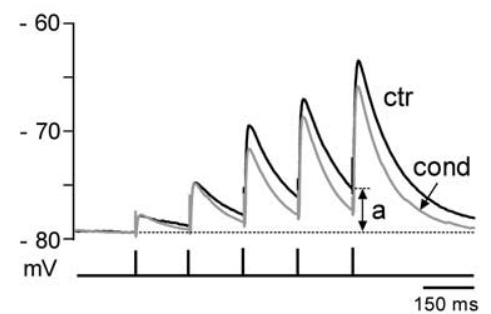

B

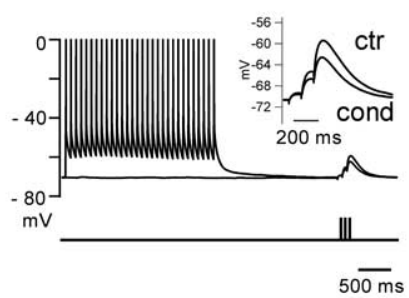

E

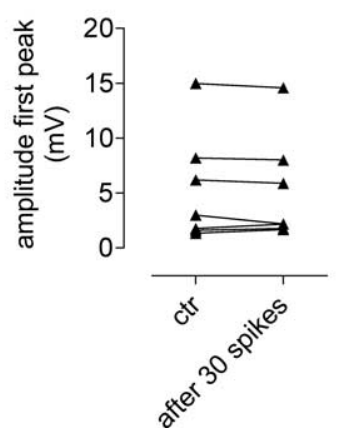

C

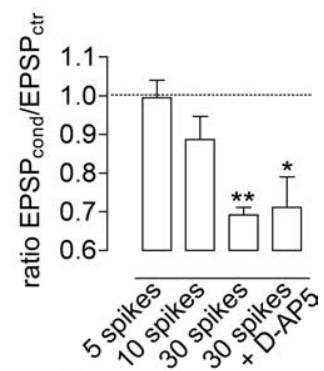

F

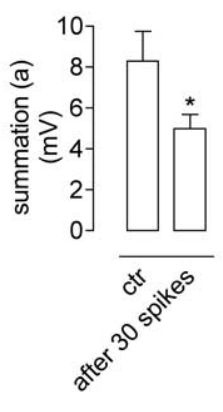

Figure 6. Depression of KAR-EPSPs can also be induced by a large train of action potentials and is important for summation properties of mossy fiber EPSPS. $\boldsymbol{A}$, Applying a train of five spikes without pairing with synaptic stimulation does not change the amplitude of KAR-EPSPs. $\boldsymbol{B}$, In contrast to what we observed with a train of five spikes, a train of 30 spikes induces a clear reduction in KAR-EPSP amplitude. C, Summary of the effects of trains of 5, 10, or 30 spikes on KAR-EPSPs. The decrease of KAR-EPSPS induced by the train of 30 spikes also occurred in the presence of $50 \mu \mathrm{M}$ D-AP5. D, Representative traces of Mf-EPSPs obtained without blocking AMPA receptors in control conditions (ctr; black) and after applying a train of 30 spikes (cond; gray). In conditions in which KAR-EPSPs are depressed by 30\%, the train of EPSPs displayed significantly less summation (measured as a) compared with the control train of EPSPS. $\boldsymbol{E}$, The train of 30 spikes did not significantly change the amplitude of the first stimulus, suggesting that this protocol did not have any presynaptic effects, nor did it affect AMPA receptors. $\boldsymbol{F}$, Summary plot of the effect of a train of 30 spikes in the summation (measured in $\boldsymbol{A}$ as indicated in $\boldsymbol{D}$ ) of EPSPs obtained after stimulating mossy fiber with a train of five

intracellular $\mathrm{Ca}^{2+}$ by synaptic NMDARs that are likely closely localized with synaptic KARs. However, because mossy fiber synapses have multiple release sites, it remains possible that a bulk increase of $\mathrm{Ca}^{2+}$ in the postsynaptic spine is required for the 
regulation of KARs. Short-term depression of KAR-EPSPs is triggered by the coordinated firing of action potentials and mossy fiber synaptic stimulation only when action potential fires during the glutamatergic EPSC. It thus represents a novel form of spiketiming-dependent plasticity (Markram et al., 1997; Dan and Poo, 2004).

Although EPSCs mediated by KARs have a much smaller amplitude than that mediated by AMPARs, they also display much slower kinetics and prominent summation properties (Castillo et al., 1997). These features allow KAR-EPSPs to play a prominent role in information transfer in CA1 interneurons by imposing a substantial depolarization during sustained afferent activity (Frerking and Ohliger-Frerking, 2002). In agreement with the important role of kainate receptors for summation properties of EPSPs (Frerking and Ohliger-Frerking, 2002), we observed that a $30 \%$ reduction of KAR-EPSPs is sufficient to induce a significant change in the summation of mossy fiber-EPSPs. This indicates that the short-term depression described here might have important physiological consequences mainly during sustained afferent activity controlling CA3 pyramidal cell depolarization. The precise role of kainate receptors in signal integration at mossy fiberCA3 pyramidal cell synapses is far from being completely understood. Nevertheless, short-term depression of KAR-EPSCs in conditions of high afferent activity leading to spike discharge is expected to exert a feedback inhibition on the sustained depolarization induced by KAR-EPSPs. Interestingly, it was reported that KAR-EPSCs show attenuated forms of presynaptic plasticity compared with AMPAR-EPSCs (Ito et al., 2004). Both mechanisms will concur in preventing the overactivation of synaptic KARs under conditions of sustained network activity and might thus favor information processing in conditions of short bursts of afferent stimulation.

\section{References}

Alger BE (2002) Retrograde signaling in the regulation of synaptic transmission: focus on endocannabinoids. Prog Neurobiol 68:247-286.

Castillo PE, Malenka RC, Nicoll RA (1997) Kainate receptors mediate a slow postsynaptic current in hippocampal CA3 neurons. Nature 388:182-186.

Cho K, Francis JC, Hirbec H, Dev K, Brown MW, Henley JM, Bashir ZI (2003) Regulation of kainate receptors by protein kinase C and metabotropic glutamate receptors. J Physiol (Lond) 548:723-730.

Contractor A, Sailer AW, Darstein M, Maron C, Xu J, Swanson GT, Heinemann SF (2003) Loss of kainate receptor-mediated heterosynaptic facilitation of mossy-fiber synapses in KA2 - / - mice. J Neurosci 23:422-429.

Coussen F, Normand E, Marchal C, Costet P, Choquet D, Lambert M, Mege RM, Mulle C (2002) Recruitment of the kainate receptor subunit glutamate receptor 6 by cadherin/catenin complexes. J Neurosci 22:6426-6436.

Coussen F, Perrais D, Jaskolski F, Sachidhanandam S, Normand E, Bockaert J, Marin P, Mulle C (2005) Co-assembly of two GluR6 kainate receptor splice variants within a functional protein complex. Neuron 47:555-566.
Dan Y, Poo MM (2004) Spike timing-dependent plasticity of neural circuits. Neuron 44:23-30.

Diana MA, Marty A (2004) Endocannabinoid-mediated short-term synaptic plasticity: depolarization-induced suppression of inhibition (DSI) and depolarization-induced suppression of excitation (DSE). Br J Pharmacol 142:9-19.

Frerking M, Ohliger-Frerking P (2002) AMPA receptors and kainate receptors encode different features of afferent activity. J Neurosci 22:7434-7443.

Ghetti A, Heinemann SF (2000) NMDA-dependent modulation of hippocampal kainate receptors by calcineurin and $\mathrm{Ca}(2+) /$ calmodulindependent protein kinase. J Neurosci 20:2766-2773.

Hirbec H, Francis JC, Lauri SE, Braithwaite SP, Coussen F, Mulle C, Dev KK, Couthino V, Meyer G, Isaac JT, Collingridge GL, Henley JM (2003) Rapid and differential regulation of AMPA and kainate receptors at hippocampal mossy fibre synapses by PICK1 and GRIP. Neuron 37:625-638.

Huettner JE (2003) Kainate receptors and synaptic transmission. Prog Neurobiol 70:387-407.

Ito K, Contractor A, Swanson GT (2004) Attenuated plasticity of postsynaptic kainate receptors in hippocampal CA3 pyramidal neurons. J Neurosci 24:6228-6236.

Kidd FL, Isaac JT (1999) Developmental and activity-dependent regulation of kainate receptors at thalamocortical synapses. Nature 400:569-573.

Lerma J (2003) Roles and rules of kainate receptors in synaptic transmission. Nat Rev Neurosci 4:481-495.

Marchal C, Mulle C (2004) Postnatal maturation of mossy fibre excitatory transmission in mouse CA3 pyramidal cells: a potential role for kainate receptors. J Physiol (Lond) 561:27-37.

Markram H, Lubke J, Frotscher M, Sakmann B (1997) Regulation of synaptic efficacy by coincidence of postsynaptic APs and EPSPs. Science 275:213-215.

Mulle C, Andreas S, Pérez-Otaño I, Dickinson-Anson H, Castillo PE, Bureau I, Maron C, Gage FH, Mann JR, Bettler B, Heinemann SF (1998) Altered synaptic physiology and reduced susceptibility to kainate induced seizures in GluR6-deficient mice. Nature 392:601-604.

Nicoll RA, Schmitz D (2005) Synaptic plasticity at hippocampal mossy fibre synapses. Nat Rev Neurosci 6:863-876.

Park Y, Jo J, Isaac JT, Cho K (2006) Long-term depression of kainate receptor-mediated synaptic transmission. Neuron 49:95-106.

Pinheiro P, Mulle C (2006) Kainate receptors. Cell Tissue Res 326:457-482.

Ruiz A, Sachidhanandam S, Utvik JK, Coussen F, Mulle C (2005) Distinct subunits in heteromeric kainate receptors mediate ionotropic and metabotropic function at hippocampal mossy fiber synapses. J Neurosci 25:11710-11718.

Salin P, Scanziani M, Malenka R, Nicoll R (1996) Distinct short-term plasticity at two excitatory synapses in the hippocampus. Proc Natl Acad Sci USA 93:13304-13309.

Tong G, Shepherd D, Jahr CE (1995) Synaptic desensitization of NMDA receptors by calcineurin. Science 267:1510-1512.

Traynelis SF, Wahl P (1997) Control of rat GluR6 glutamate receptor open probability by protein kinase A and calcineurin. J Physiol (Lond) 503:513-531.

Vignes M, Collingridge GL (1997) The synaptic activation of kainate receptors. Nature 388:179-182.

Zucker RS, Regehr WG (2002) Short-term synaptic plasticity. Annu Rev Physiol 64:355-405. 